This article is licensed under the Creative Commons Attribution-NonCommercial 4.0 International License (CC BY-NC) (http://www.karger.com/Services/OpenAccessLicense). Usage and distribution for commercial purposes requires written permission.

\title{
A Case Report Demonstrating the Potential Clinical Benefit of Exhaustive Molecular Profiling in an Aggressive Muscle-Invasive High-Grade Metastatic Urothelial Carcinoma
}

Amaya Ramírez de Olano ${ }^{a} \quad J o a q u i m ~ B e l l m u n t^{a} \quad$ Ana Rodrigo $^{\text {b, c }}$ Luis Álvarez $^{b-d} \quad$ Adriana Terrádez $^{\text {b, c }}$ Jesús García-Foncillas ${ }^{b, e}$ Jean-François Laes ${ }^{c}$

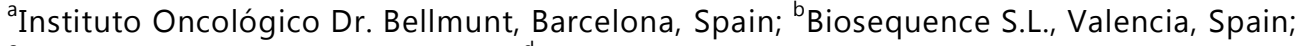
'OncoDNA SA, Gosselies, Belgium; ${ }^{d}$ I3S Instituto de Investigação e Inovação da Universidade do Porto, Porto, Portugal; ${ }^{e}$ Institute University Hospital "Fundación Jiménez Díaz", Madrid, Spain

\section{Keywords}

Urothelial carcinoma $\cdot$ RB1 $\cdot$ TOP2A $\cdot$ TUBB3 $\cdot$ Adriamycin $\cdot$ Abraxane $\cdot$ Next-generation sequencing $\cdot$ Immunohistochemistry

\section{Abstract}

We present a muscle-invasive high-grade metastatic urothelial carcinoma patient, aged 71 years, with rapid progression from the diagnosis and a poor prognosis after 3 lines of treatment. A clinical exhaustive genomic profile was performed with the goal of finding potential actionable molecular alterations. The patient showed significant symptomatic and 


\section{Case Reports in Oncology}

laboratory improvement with a nonstandard chemotherapy combination treatment identified by the molecular profiling, which would otherwise not have been considered. This approach illustrates the clinical benefit of a comprehensive genomic analysis in an aggressive and refractory urothelial carcinoma.

(C) 2017 The Author(s)

Published by S. Karger AG, Basel

\section{Introduction}

Bladder cancer (BC) is one of the most common tumors worldwide, becoming one of the main causes of tumor-related deaths. According to worldwide estimations, 430,000 cases and 165,000 deaths of urothelial bladder cancer (UBC) occurred in 2012, ranking it the 9th most common type of cancer for both genders, 6th in males and 19th in females, with a worldwide sex ratio of 3.5:1. UBCs are more common in developed countries, with $60 \%$ of all incident cases being the higher rates in males detected in Western and Southern Europe, particularly in Spain (regional data: age-standardized rate $=36.7$ per 100,000). Female rates are highest in Northern America and Western Europe [1].

Mortality rates are much lower than the incidence rates, with the highest agestandardized rate estimated in Western Asia in men (8.4 per 100,000) and in Northern Africa in women (1.6). Overall, BC mortality has been decreasing all over the world except in countries undergoing rapid economic transition [2].

Approximately $75 \%$ of newly diagnosed UBCs are noninvasive and have a high rate of recurrence and progression despite local therapy. The remaining $25 \%$ of newly diagnosed UBCs present with muscle invasion and need either radical surgery or radiotherapy but often still have poor outcomes despite systemic therapy $[3,4]$.

At the population level, there is clear evidence of tobacco smoking as the main risk factor for BC development, estimated to account for $50 \%$ of tumors. Estimated pooled values of relative risk (RR) in cohort and case-control studies are 3.37 in current and 1.98 in former smokers. Moreover, both current smokers (RR: 1.89) and former smokers (RR: 1.66) present a higher risk of BC mortality compared with never-smokers [5].

Besides tobacco consumption, it has been suggested that $20 \%$ of all UCBs are related to occupational exposure to carcinogens, namely aromatic amines, polycyclic aromatic hydrocarbons, and chlorinated hydrocarbons, which is the second most important risk factor. Other risk factors that are commonly related to UBC are genetic susceptibility, with a growing knowledge of the inherited genetic factors; dietary factors, mainly related to fluid intake; environmental pollution, exposure to arsenic in drinking water has been recognized as a cause of UBC; medical conditions, associated with a high predisposition to bladder tumorigenesis as chronic urinary retention and upper tract dilation that increase urothelial exposure to carcinogens and carcinogenesis associated with chronic inflammation or schistosomiasis; moreover, gender, race, and socioeconomic status are also among the considered factors [6].

According to the molecular genetic evidence, high-grade invasive $\mathrm{BC}$ is characterized by alterations in the tumor suppressor genes, p53 and retinoblastoma (RB1) pathways. These genes interact with the Ras-mitogen-activated protein kinase (MAPK) signal transduction pathways [7]. 


\section{Case Reports in Oncology}

We report the case of a patient with muscle-invasive high-grade metastatic urothelial carcinoma presenting a quick progression from the diagnosis and a poor prognosis after 3 lines of treatment. A clinical exhaustive genomic profile (OncoDEEP ${ }^{\circledR}$ Clinical Plus) was requested, with the goal of finding potential actionable molecular alterations. Based on TOP2A and TUBB3 expression patterns, the patient was started on Adriamycin/Abraxane treatment, achieving a great clinical benefit.

\section{Case Report}

The patient is a 71-year-old man, former smoker, with a history of dyslipidemia, hypertension, and well-controlled diabetes mellitus type 2 .

In July 2014, he debuted clinically with hematuria with a diagnosis of muscle-infiltrating bladder tumor. The patient underwent an urethrocystoscopy and transurethral resection of the bladder. Pathology revealed a high-grade papillary urothelial carcinoma, which infiltrated the subepithelial connective tissue and fibers of the smooth muscle fibers of the muscular layer itself. He was considered to have PT2G3. The patient was treated with 4 cycles of neoadjuvant chemotherapy cisplatin-gemcitabine. In November 2014, a cystectomy was performed. Pathology revealed a high-grade urothelial carcinoma of $2 \mathrm{~cm}$ with infiltration of perivesical fat that showed vascular and lymphatic invasion, with negative margins and $0 / 6$ obturator and iliac lymph nodes. He was considered to have PT3aG3N0M0.

In May 2015, the patient presented with a nodule on the dorsal side of the penis. PET/CT confirmed a unique hypermetabolic tumor on the glans (SUVmax: 6), without other localizations of disease. A partial phallectomy with the range extended to radical affection was undertaken. The pathology revealed a high-grade metastatic urothelial carcinoma of $4 \mathrm{~cm}$ in diameter, which infiltrated the balanopreputial sulcus, glans, and cavernous bodies and showed vascular and perineural invasion. The patient started treatment with 4 cycles of carboplatin-paclitaxel until October 2015.

In December 2015, a CT scan of the chest and abdomen revealed multiple nodules in the lung and liver. A PET/CT confirmed the relapse revealing multiple new bilobar liver lesions, mesorectal fascia implantation, and bone and bilateral pulmonary metastases (Fig. 1). The patient had pain in the right ischium and was treated with 10 sessions of analgesic radiotherapy until February 2016 with analgesic effect and chemotherapy regimen consisting of vinflunine $280 \mathrm{mg} / \mathrm{m}^{2}+$ Zometa. After the second cycle, the treatment was suspended due to hepatic progression confirmed by a new PET/CT.

At this point, exhaustive genomic profiling of the tumor was performed (Biosequence S.L., Valencia, Spain) using an OncoDEEP ${ }^{\circledR}$ Clinical Plus solution (OncoDNA SA, Gosselies, Belgium) to obtain outcomes about its sensitivity to different types of treatment (target therapy, chemotherapy, and immunotherapy agents) and recruiting clinical trials. Paraffinembedded tumor tissue was used to analyze 409 genes employing next-generation sequencing (NGS) (Ion Torrent Technology; Life Technologies, Carlsbad, CA, USA) in combination with immunohistochemistry (IHC) studies. The results of the NGS analysis showed the presence of 107 variants including 4 actionable mutations (FANCA S1088F, HIF1A P582S, CSMD3 E1883*, and RB1 S576*). IHC revealed high expression of the p4EBP1, ERCC1, and RRM1 genes; positive expression of the TS, TOP2A, and HER2 genes; low expression of PD-L1 and 


\section{Case Reports in Oncology}

TUBB3; and negative expression of the CD8 gene. NGS and IHC results are summarized in Table 1. Treatment with anthracyclines or taxanes was suggested as therapy associated with a potential clinical benefit based on the TOP2A and TUBB3 expression patterns, respectively.

An echocardiogram was performed, which showed a slightly hypertrophic left ventricle with preserved systolic function and ejection fraction of 74\%. The patient presented ECOG 1 and $80 \%$ IK. Due to the need for a rapid response, the patient was considered a candidate for a combination therapy with anthracyclines and taxanes, according to the molecular profiling outcome. In March 2016, the patient started treatment with Adriamycin $60 \mathrm{mg} / \mathrm{m}^{2}$ (day 1) + Abraxane $100 \mathrm{mg} / \mathrm{m}^{2}$ (days 1 and 8) every 21 days.

Some days after initiating treatment, the patient felt significantly better and showed further improvement over the following weeks. After 3 cycles of treatment, a control CT scan indicated partial regression of the pulmonary and liver metastases. After 6 cycles, a PET/CT revealed a significant decrease in the largest hepatic lesion $(26 \times 12 \mathrm{~mm}$ to $49 \times 30 \mathrm{~mm}$, previously $75 \mathrm{~mm}$ ) (Fig. 2).

The patient completed 8 cycles in September 2016, maintaining response until December 2016. Currently, 6 months after the chemotherapy ended, the patient presents pain on palpation in the right hypochondrium suspicious of progression. The results of PET/CT are pending in order to assess the following treatment.

\section{Discussion}

The patient presented with a progressive cancer and rapid deterioration of his performance status after receiving 3 standard lines of treatment. The standard of treatment of muscle-invasive and metastatic $\mathrm{BC}$ is limited: the first-line treatment for patients must be based on a cisplatin-containing combination. For the second line, since chemotherapy agents tested in phase 2 studies have not proven a global survival benefit [8], vinflunine is the only drug approved for use by the EMA $[9,10]$.

At this point, the need for a personalized treatment was raised. Given the remarkable advances in the identification of molecular biomarkers, we decided to perform exhaustive tumor molecular profiling [11].

Mutations detected in the molecular analysis of the tumor were relevant for showing the presence of the likely driver of malignancy involving the RB1 gene. The variant detected (p.S576*) induces a premature stop codon within the pocket domain of the protein required for its binding to the threonine-phosphorylated domain $\mathrm{C}$, thereby not preventing its interaction with heterodimeric E2F/DP transcription factor complexes required for its tumor suppressor activity [12].

RB1, a tumor suppressor gene, is a negative regulator of the cell cycle, and its alterations are related to carcinogenesis in several cancers. For muscle-invasive $\mathrm{BC}$, the expression loss of RB1 has been described to be an adverse prognosis biomarker [13]. In addition, those BCs presenting a mutation of the RB1 gene combined with low FGFR3 levels have been associated with a significantly poor disease-specific survival [14]. These findings are compatible with the evolution of the patient presenting a rapid progression from the diagnosis and a poor prognosis after 3 lines of treatment. 


\section{Case Reports in Oncology}

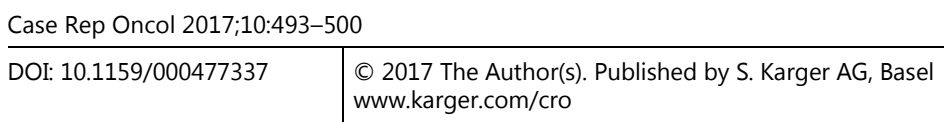

Ramírez de Olano et al.: Clinical Benefit of Exhaustive Molecular Profiling in a Patient with an Aggressive Urothelial Carcinoma

The IHC analysis was remarkable for identifying the potential clinical benefit of treatment with anthracycline and taxane chemotherapies. Despite the fact that there were no response studies with this combination of chemotherapies in this particular scenario, the patient received Adriamycin/Abraxane. It is worth mentioning that we would never have considered these specific chemotherapy combinations in patients with these features without the molecular insights. The patient responded for 8 months and he has kept responding for 5 months, even though he had a much shorter life expectancy.

This case demonstrates the major utility of comprehensive tumor molecular profiling, combining NGS and IHC analysis, in the case of aggressive BC. The Adriamycin/Abraxane chemotherapy combination needs to be further investigated in future patients and/or clinical studies.

\section{Statement of Ethics}

The authors have no ethical conflicts to disclose.

\section{Disclosure Statement}

The authors declare that they have no competing interests and no conflicts of interest.

\section{References}

1 Ferlay J, Soerjomataram I, Dikshit R, Eser S, Mathers C, Rebelo M, et al: Cancer incidence and mortality worldwide: sources, methods and major patterns in GLOBOCAN 2012. Int J Cancer 2015;136:E359E386.

2 Antoni S, Ferlay J, Soerjomataram I, Znaor A, Jemal A, Bray F: Bladder cancer incidence and mortality: a global overview and recent trends. Eur Urol 2017;71:96-108.

3 Babjuk M, Oosterlinck W, Sylvester R, Kaasinen E, Böhle A, Palou-Redorta J, et al: EAU Guidelines on Non-Muscle-Invasive Urothelial Carcinoma of the Bladder, the 2011 Update. Eur Urol 2011;59:9971008.

4 Stenzl A, Cowan NC, De Santis M, Kuczyk MA, Merseburger AS, Ribal MJ, et al: Treatment of muscleinvasive and metastatic bladder cancer: update of the EAU guidelines. Eur Urol 2011;59:1009-1018.

5 Cumberbatch MG, Rota M, Catto JWF, La Vecchia C: The role of tobacco smoke in bladder and kidney carcinogenesis: a comparison of exposures and meta-analysis of incidence and mortality risks. Eur Urol 2016;70:458-466.

-6 Burger M, Catto JWF, Dalbagni G, Grossman HB, Herr H, Karakiewicz P, et al: Epidemiology and risk factors of urothelial bladder cancer. Eur Urol 2013;63:234-241.

7 Netto GJ: Molecular biomarkers in urothelial carcinoma of the bladder: are we there yet? Nat Rev Urol 2011;9:41-51.

8 Oing C, Rink M, Oechsle K, Seidel C, von Amsberg G, Bokemeyer C: Second line chemotherapy for advanced and metastatic urothelial carcinoma: vinflunine and beyond-a comprehensive review of the current literature. J Urol 2016;195:254-263.

$\$ 9$ Bellmunt J, Théodore C, Demkov T, Komyakov B, Sengelov L, Daugaard G, et al: Phase III trial of vinflunine plus best supportive care compared with best supportive care alone after a platinumcontaining regimen in patients with advanced transitional cell carcinoma of the urothelial tract. J Clin Oncol 2009;27:4454-4461. 


\section{Case Reports in Oncology}

Ramírez de Olano et al.: Clinical Benefit of Exhaustive Molecular Profiling in a Patient with an Aggressive Urothelial Carcinoma

-10 Lázaro M, Gallardo E, Doménech M, Pinto Á, Del Alba AG, Puente J, et al: SEOM Clinical guideline for treatment of muscle-invasive and metastatic urothelial bladder cancer (2016). Clin Transl Oncol 2016;18:1197-1205.

-11 Ross JS, Wang K, Khaira D, Ali SM, Fisher HAG, Mian B, et al: Comprehensive genomic profiling of 295 cases of clinically advanced urothelial carcinoma of the urinary bladder reveals a high frequency of clinically relevant genomic alterations. Cancer 2016;122:702-711.

$\checkmark 12$ Burke JR, Hura GL, Rubin SM: Structures of inactive retinoblastoma protein reveal multiple mechanisms for cell cycle control. Genes Dev 2012;26:1156-1166.

13 Shariat SF, Tokunaga H, Zhou J, Kim J, Ayala GE, Benedict WF, et al: p53, p21, pRB, and p16 expression predict clinical outcome in cystectomy with bladder cancer. J Clin Oncol 2004;22:1014-1024.

14 Lindgren D, Sjödahl G, Lauss M, Staaf J, Chebil G, Lövgren K, et al: Integrated genomic and gene expression profiling identifies two major genomic circuits in urothelial carcinoma. PLoS One 2012;7:e38863.

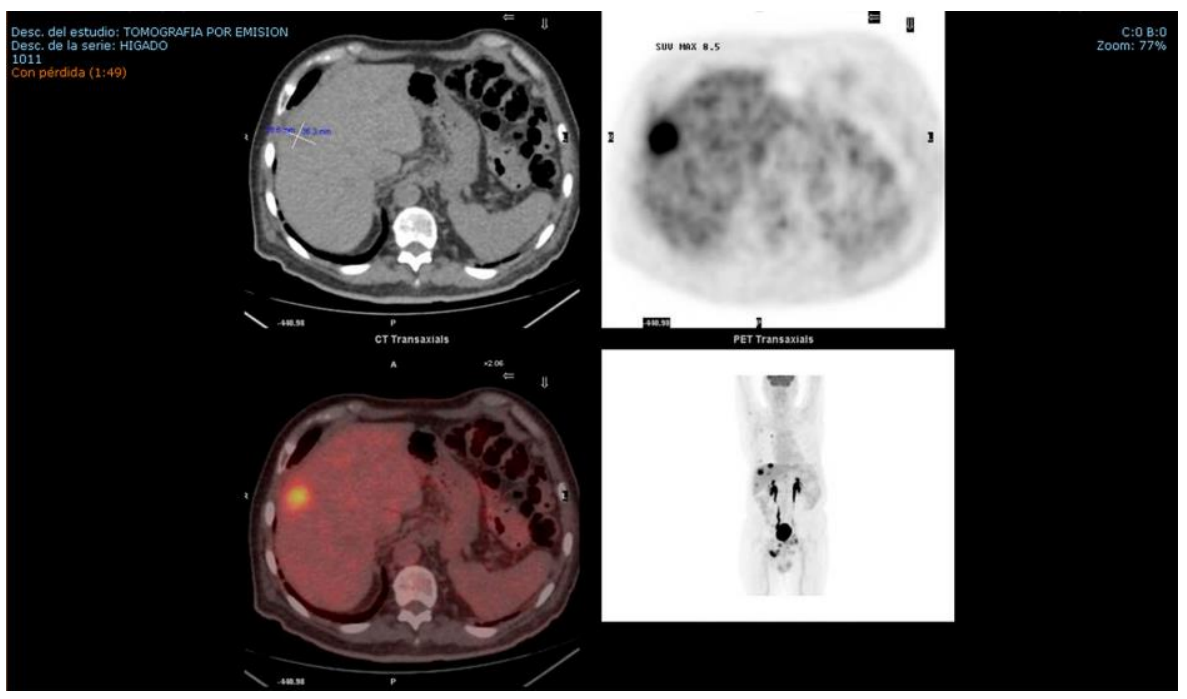

Fig. 1. PET/CT confirming the relapse in February 2016. 


\section{Case Reports in Oncology}

Case Rep Oncol 2017:10:493-500

DOI: $10.1159 / 00047733$

(C) 2017 The Author(s). Published by S. Karger AG, Basel www.karger.com/cro

Ramírez de Olano et al.: Clinical Benefit of Exhaustive Molecular Profiling in a Patient with an Aggressive Urothelial Carcinoma

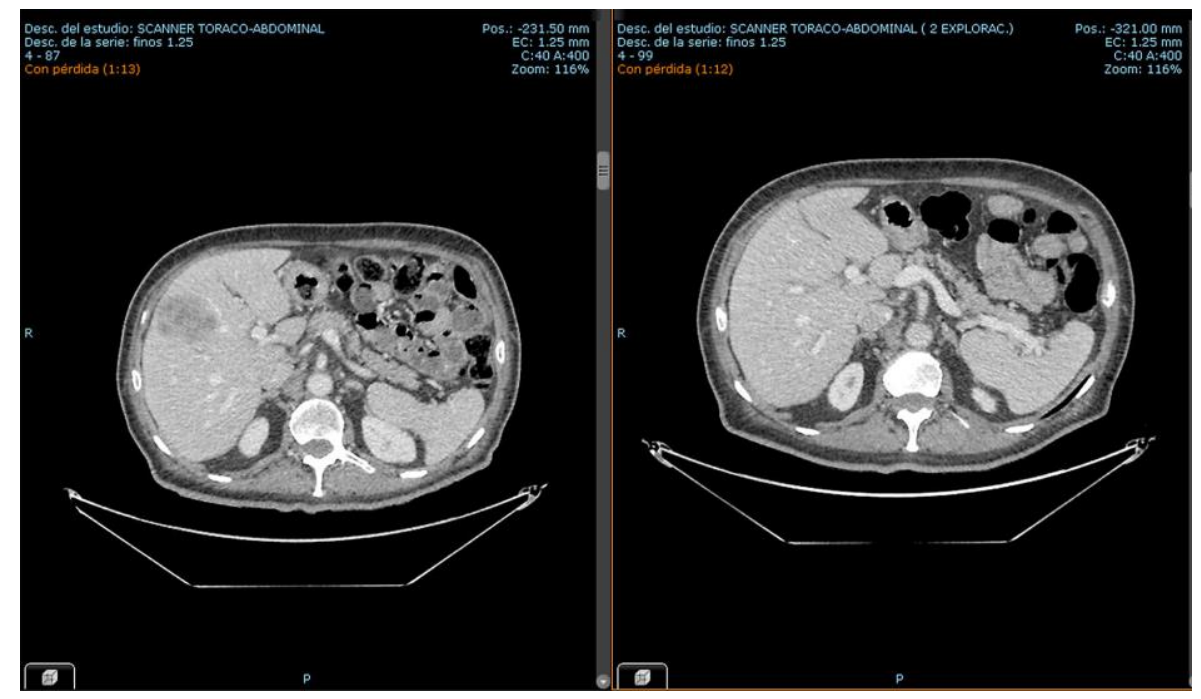

Fig. 2. CT images from the hepatic lesion before (March 2016) and after treatment (August 2016). 


\section{Case Reports in Oncology}

Case Rep Oncol 2017;10:493-500 \begin{tabular}{l|l}
\hline DOI: $10.1159 / 000477337$ & $\begin{array}{l}\text { C } 2017 \text { The Author(s). Published by S. Karger AG, Basel } \\
\text { www.karger.com/cro }\end{array}$
\end{tabular}

Ramírez de Olano et al.: Clinical Benefit of Exhaustive Molecular Profiling in a Patient with an Aggressive Urothelial Carcinoma

Table 1. Results of the exhaustive genomic profiling (NGS and IHC) of the tumor (OncoDEEP ${ }^{\circledR}$ Clinical Plus)

NGS

\begin{tabular}{|c|c|c|}
\hline \multicolumn{2}{|l|}{ Variants } & Results \\
\hline \multicolumn{2}{|c|}{ Actionable $(n=4)$} & FANCA p.S1088F; CSMD3 p.E1883*; RB1 p.S576*; HIF1A p.P582S \\
\hline \multicolumn{2}{|c|}{$\begin{array}{l}\text { Unknown significance } \\
(n=48)\end{array}$} & $\begin{array}{l}\text { PDE4DIP p.L1727P; FANCD2; ROS1; EML4; SMARCA4 p.K1439E; DDR2; EPHA7 p.P805L; NOTCH1 } \\
\text { p.P1405H; ETV4; ETV4; KMT2D p.S4010P; ASXL1 p.S915A; UBR5 p.H757P; TSC1; LIFR p.P961Q; LPP } \\
\text { p.I512L; MN1 p.K287Q; DAXX p.A358P; NLRP1 p.A1152T; MAML2 p.I480M; PBRM1 p.G936A; PIK3R1 } \\
\text { p.P617A; TBX22 p.S67P; TBX22 p.S69A; AURKB p.D289A; FBXW7 p.V37G; CHEK1 p.D329E; TGM7; } \\
\text { NOTCH2 p.P306T; WRN p.P1077L; WT1 p.S6P; AURKB p.T35P; KMT2A p.S2740R; IGF1R p.T207P; } \\
\text { IGF1R p.Q649L; NLRP1 p.V902L; UGT1A1 p.V261L; LPP p.L81F; NOTCH4 p.T684M; PKHD1; FANCC } \\
\text { p.S5L; PTCH1 p.P299L; TET1 p.L1346S; TGM7 p.R216L; ERCC4 p.E875G; MARK4 p.P518L; TIMP3 } \\
\text { p.P201L; AMER1 p.G954E }\end{array}$ \\
\hline \multicolumn{2}{|c|}{$\begin{array}{l}\text { Probably polymorphism } \\
(n=52)\end{array}$} & $\begin{array}{l}\text { IKBKE p.P713L; ALK p.D1529E; LTF p.K47R; TNK2 p.P725L; SDHA p.V657I; MTRR p.I49M; ROS1 } \\
\text { p.R167Q; SYNE1 p.G8323A; SYNE1; ETV1 p.S100G; SAMD9 p.I143T; XPA; BUB1B p.R349Q; PER1 } \\
\text { p.A962P; MBD1 p.P345A; AURKA p.I57V; EML4 p.K398R; CSMD3 p.I219M; NIN p.P1111A; THBS1 } \\
\text { p.N700S; TGFBR2; DST; sep09; PLEKHG5 p.T315S; XPC p.A499V; SAMD9 p.V549L; RNF213 p.M270T; } \\
\text { RNF213 p.V3838L; ERCC2 p.D312N; MYH9 p.I1626V; IDH1 p.V178I; XPC; TSC1 p.M322T; RNF213 } \\
\text { p.H4691R; SYNE1 p.T8687I; FANCA; FANCA; FANCA p.P643A; FANCA p.A412V; MTR p.D314N; RNF213 } \\
\text { p.P729L; RNF213 p.K1034M; PIK3CG p.S442Y; MAP3K7; CYP2C19 p.V331I; ERCC5 p.C529S; PKHD1 } \\
\text { p.T579M; STK36 p.K295R; STK36 p.R1112Q; STK36 p.R477W; PMS2 p.T511A; PTPRT p.L761V }\end{array}$ \\
\hline \multicolumn{2}{|c|}{$\begin{array}{l}\text { Medically actionable } \\
\text { incidental findings }(n=3)\end{array}$} & THBS1 p.N700S; FANCA p.S1088F; HIF1A p.P582S \\
\hline \multicolumn{3}{|l|}{ IHC } \\
\hline \multicolumn{3}{|l|}{ biomarker } \\
\hline PTEN & Positive & Potential lack of clinical benefits of PI3K/mTOR inhibitors \\
\hline p4EBP1 & High & Potential lack of clinical benefits of PI3K/mTOR inhibitors \\
\hline TS & Positive & Potential lack of clinical benefits of 5-FU-based chemotherapy \\
\hline TOP2A & Positive & Potential clinical benefits of anthracycline-based chemotherapy \\
\hline PD-L1 & Low & Potential lack of clinical benefits of PD-1/PD-L1 inhibitors \\
\hline CD8 & Negative & Potential lack of clinical benefits of PD-1/PD-L1 inhibitors \\
\hline ERCC1 & High & Potential lack of clinical benefits of platinum-based chemotherapy \\
\hline HER2 & Positive & Indeterminate clinical benefit \\
\hline RRM1 & High & Potential lack of clinical benefits of gemcitabine \\
\hline TUBB3 & Low & Potential clinical benefits of taxane-based chemotherapy \\
\hline
\end{tabular}

